\title{
ANÁLISIS DEL FUNCIONAMIENTO DE LA MEMORIA OPERATIVA EN NIÑOS CON TRASTORNOS EN EL APRENDIZAJE
}

\author{
Tulia Ocampo Gaviria ${ }^{1 *}$, Óscar Sierra Fitzgerald ${ }^{1 * *}$ \\ ${ }^{1}$ Instituto de Psicología, Universidad del Valle, Cali - Colombia
}

\author{
Recibido, diciembre 17/2013 \\ Concepto de evaluación, abril 24/2014 \\ Aceptado, mayo 28/2014
}

\begin{abstract}
Referencia: Ocampo Gaviria, T. y Sierra Fitzgerald, O. (2014).Análisis del Funcionamiento de la memoria operativa en niños con trastornos en el aprendizaje. Acta Colombiana de Psicología, 17 (2), pp 81-90. DOI:10.14718/ACP.2014.17.2.9
\end{abstract}

Resumen

\begin{abstract}
La presente investigación buscó indagar la manera en que los diferentes componentes del modelo de memoria operativa, inicialmente propuesto por Baddeley y Hitch en 1974, están afectados en dos tipos de trastornos en el aprendizaje escolar en niños de siete años: Trastorno en el Aprendizaje de la Lectura (TAL) y trastorno concomitante de TAL con Trastorno en el Aprendizaje de las Matemáticas (TAL/TAM), con el fin de ayudar a comprender las diferencias entre los diferentes trastornos en el aprendizaje escolar. En la investigación se estudiaron 45 niños: 15 niños sin Trastornos Específicos en el Aprendizaje (Sin TEA), 15 con TAL y 15 con TAL/TAM. Los resultados muestran que los niños con cualquiera de estos trastornos específicos del aprendizaje presentan déficits en la memoria operativa, y que éste es mayor en aquellos que presentan un trastorno más generalizado en el aprendizaje como es el caso de los niños con TAL/TAM, confirmándose la relación estrecha entre la memoria operativa y el aprendizaje escolar. Adicionalmente, se encontró que los niños con TAL presentaban dificultades significativas en la memoria operativa verbal cuando se requiere no sólo el almacenamiento de la información, sino también su procesamiento. Los niños con TAL/TAM se diferenciaron de los niños con TAL en tareas de memoria operativa viso-espacial, pudiendo concluirse que un déficit en esta habilidad cognoscitiva es lo que los pondría en riesgo de presentar dificultades en matemáticas, además de las de lectura.

Palabras clave: Memoria operativa, aprendizaje escolar, trastornos en el aprendizaje
\end{abstract}

\section{ANALYSIS OF WORKING MEMORY FUNCTIONING IN CHILDREN WITH LEARNING DISORDERS}

\begin{abstract}
The present research study sought to examine the way in which the different components of the working memory model initially proposed by Baddeley and Hitch in 1974 are affected in two types of learning disorders in seven year old children: Reading Learning Disorder (TAL for its Spanish acronym) and concomitant Reading and Mathematics Learning Disorder (TAL/TAM, for its Spanish acronym), in order to achieve greater understanding of the differences between the various school learning disorders. 45 children were assessed in this research: 15 children without Specific Learning Difficulties (Sin TEA, for its Spanish acronym), 15 with TAL and 15 with TAL/TAM. Results show that children with either of these specific learning disorders have deficits in working memory and that these are greater in those children who present a more generalized learning disorder, as in the case of the children with TAL/TAM, confirming the close relationship between working memory and school learning. In addition, it was found that children with TAL have significant difficulties in verbal working memory, when not only information storage but also its processing is requiered. Children with TAL/TAM differed from those with TAL in visuospatial working memory tasks, leading to the conclusion that a deficit in this cognitive ability is what would put them at risk for difficulties in mathematics, in addition to reading problems.

Key words: Working memory, school learning, learning disorders
\end{abstract}

* Tulia Ocampo Gaviria B.A., M.Ed., M.S. Dirección para correspondencia: Calle 4 No.27-104, Cali, Colombia. tocampogav@gmail.com ** Esta investigación fue dirigida por Óscar Sierra Fitzgerald, M.S., Ph.D., Neuropsicólogo Clínico, Profesor Titular del Instituto de Psicología de la Universidad del Valle, fallecido antes de la publicación del presente artículo.

La autora agradece a la Psicóloga Katherine Mancilla por su contribución en la recolección de datos de esta investigación y al Profesor Gabriel Arteaga Díaz del Instituto de Psicología de la Universidad del Valle por su apoyo en la edición de este documento, en ausencia del Profesor Sierra. 


\title{
ANÁLISE DO FUNCIONAMENTO DA MEMÓRIA OPERACIONAL EM CRIANÇAS COM TRANSTORNOS DE APRENDIZAGEM
}

\begin{abstract}
Resumo
A presente pesquisa procurou indagar a maneira em que os diferentes componentes do modelo de memória operacional, inicialmente proposto por Baddeley e Hitch em 1974, estão afetados em dois tipos de transtornos na aprendizagem escolar em crianças de sete anos: Transtorno na Aprendizagem da Leitura (TAL) e transtorno concomitante de TAL com Transtorno na Aprendizagem de Matemática (TAL/TAM), com objetivo de ajudar a compreender as diferenças entre os diferentes transtornos na aprendizagem escolar. Na pesquisa estudaram-se 45 crianças: 15crianças sem Transtornos Específicos na Aprendizagem (Sin TEA), 15 com TAL e 15 com TAL/TAM. Os resultados mostram que as crianças com qualquer desses transtornos específicos da aprendizagem apresentam déficits na memória operacional, e que ele é maior naqueles que apresentam um transtorno mais generalizado na aprendizagem como é caso das crianças com TAL/TAM, confirmando-se a relação estreita entre a memória operacional e a aprendizagem escolar. Além disso, encontrou-se que as crianças com TAL apresentavam dificuldades significativas na memória operacional verbal quando se requer não só o armazenamento da informação, mas também seu processamento. As crianças com TAL/TAM se diferenciaram das crianças com TAL em tarefas de memória operacional viso-espacial, podendo concluir-se que um déficit nesta habilidade cognoscitiva é o que os poria em risco de apresentar dificuldades em matemática, além das de leitura.

Palavras chave: Memoria operacional, aprendizagem escolar, transtornos na aprendizagem
\end{abstract}

\section{INTRODUCCIÓN}

Los trastornos en el aprendizaje pueden afectar de manera negativa el desarrollo de los niños, tanto a nivel cognoscitivo como emocional. Por lo tanto, es necesario detectar de manera temprana a los niños que empiezan a evidenciar dificultades significativas en el aprendizaje desde el inicio de su escolaridad. Para este fin es necesario partir de un modelo teórico con respaldo empírico, en el que se consideren los aspectos neurocognoscitivos, que permitan explicar estos problemas. De esta manera se podría prevenir el fracaso escolar en algunos casos, mientras que en otros se podrían reducir sus efectos negativos proveyendo intervenciones oportunas que tengan en cuenta sus fortalezas y debilidades cognoscitivas.

La teoría evolutiva de Geary $(2003,2005,2007)$ plantea que el sistema ejecutivo tiene un rol importante en el aprendizaje, y por ello incorpora el modelo de memoria operativa propuesto por Baddeley y Hitch (1974). Este modelo se basa en una estructura de multicomponentes, $y$ se ha modificado para adaptarse a las evidencias, tanto de estudios cognoscitivos, como neurocientíficos (Baddeley \& Hitch, 1994; Baddeley, 2000, 2002, 2003). El modelo define la memoria operativa como un sistema de capacidad limitada cuya función es mantener, manipular y almacenar de manera transitoria la información necesaria para la ejecución de actividades cognoscitivas complejas como la lectura, el cálculo, el razonamiento, la comprensión y el aprendizaje. Por otro lado, este sistema favorece los procesos de pensamiento humano, vinculando procesos como la percepción, la memoria a largo plazo y la acción.

En un principio el modelo de Baddeley y Hitch (1974) propuso tres componentes: un Ejecutivo Central (EC), que sirve de control atencional limitado, y de dos sistemas subordinados: el Bucle Fonológico y la Agenda Viso-Espacial, que trabajan de manera integrada con el EC. Según el modelo, el bucle fonológico almacena de manera temporal la información verbal-acústica, lo cual permite mantener la representación sonora de las palabras. La agenda viso-espacial, por su lado, retiene transitoriamente representaciones de información visual y espacial, permitiendo la orientación espacial y la solución de problemas viso-espaciales. El modelo se fue ajustando a los hallazgos de las investigaciones y se propusieron subcomponentes tanto para el bucle fonológico (un Almacén Fonológico y un Repaso Articulatorio) como para la agenda viso-espacial, uno visual y otro espacial (Baddeley, 2002, 2003; Logie, 1995). Adicionalmente, se han sugerido subprocesos en el funcionamiento del EC. Por último, se ha añadido un nuevo componente: el Búfer Episódico, un sistema que permite integrar diferentes fuentes de información, incluyendo las del bucle fonológico, de la agenda viso-espacial y de la memoria a largo plazo (Baddeley, 2000). (Para más información, véase Sierra-Fitzgerald \& Ocampo-Gaviria, 2013).

Diversos estudios han mostrado la relación entre la memoria operativa y el aprendizaje escolar, incluyendo el aprendizaje de las matemáticas y de la lectura. Varios de éstos se han fundamentado en el modelo de memoria operativa inicialmente propuesto por Baddeley y Hitch en 1974. Sierra-Fitzgerald \& Ocampo-Gaviria (2013) han clasificado las investigaciones realizadas en niños en cuatro grupos, haciendo una discusión extensa de cada uno de ellos. El primer grupo está conformado por aquellos estudios que han explorado la validez de la estructura de multicomponentes del modelo de memoria operativa desde los cuatro años. 
El segundo grupo incluye las investigaciones longitudinales que valoran la memoria operativa a edad temprana y, más adelante, el desempeño académico, con el fin de establecer si la habilidad de la memoria operativa permite predecir el rendimiento escolar. Pertenecen al tercer grupo aquellas investigaciones realizadas con muestras representativas de la población escolar, las cuales buscan correlacionar el rendimiento escolar con la ejecución en tareas de memoria operativa. Por último, en el cuarto grupo se incorporan los estudios con niños que presentan diversos Trastornos Específicos en el Aprendizaje (TEA), y que determinan si los diferentes trastornos se asocian con un perfil específico de fortalezas y debilidades en los distintos componentes del modelo. La presente investigación se enfoca en este último grupo de estudios, los relacionados con el funcionamiento de la memoria operativa en niños con diversos TEA.

Numerosos estudios muestran que niños con Trastornos en el Aprendizaje de la Lectura (TAL) pueden evidenciar déficits en todos los componentes del modelo de memoria operativa de Baddeley y Hitch (1974), en especial en el bucle fonológico y el EC con información verbal, demostrando falta de capacidad para almacenar y procesar información verbal de manera simultánea (Baqués \& Sáiz, 1999; Gathercole, Alloway, Willis \& Adams, 2006; Pickering, 2006; Swanson, 2003). Por otro lado, no hay claridad respecto a una deficiencia en la agenda viso-espacial. Hay que anotar que ninguno de los estudios utiliza medidas de EC con información viso-espacial por no contarse con tareas confiables y válidas.

Los estudios que se enfocan en los Trastornos en el Aprendizaje de las Matemáticas (TAM) evidencian que el déficit principal se observa en el EC, aunque también pueden presentar deficiencias en el bucle fonológico y en la agenda viso-espacial (Geary, Hoard, Byrd-Craven, Nugent \& Numtee, 2007; McLean \& Hitch, 1999; Miranda-Casas, Meliá-DeAlba, Marco-Taverner, Roselló \& Mulas, 2006; Passolunghi \& Siegel, 2004). Sin embargo, las investigaciones muestran que los déficits en el bucle fonológico sólo son evidentes cuando se trata de contenido numérico, mientras que en la agenda viso-espacial la dificultad se presenta principalmente en tareas del componente espacial, pero no del componente visual.

Algunas investigaciones recientes sobre la relación entre la memoria operativa y los trastornos en el aprendizaje han buscado identificar los perfiles diferenciados de fortalezas y debilidades en la memoria operativa para los diferentes trastornos del desarrollo. Entre estos se encuentra el de Pickering (2006), el cual compara niños con dislexia con aquellos que presentan un trastorno del desarrollo de la coordinación motora (dispraxia). Este estudio muestra que los niños con dislexia evidencian una debilidad en el bucle fonológico y en el EC con información verbal, mientras que los niños con trastorno de la coordinación motora presentan debilidad en la agenda viso-espacial. Tanto Pickering y Gathercole (2004) como Alloway y colaboradores (Alloway \& Temple, 2007; Alloway \& Archibald, 2008; Alloway, Rajendran \& Archibald, 2009) también comparan los perfiles de memoria operativa de niños con diferentes trastornos del desarrollo, encontrando diferencias en los diferentes componentes del modelo de memoria operativa de Baddeley y Hitch (1974).

De acuerdo con la revisión de la literatura, ninguna de estas investigaciones ha comparado niños con TAL con aquellos que presentan un trastorno concomitante en la lectura y las matemáticas (TAL/TAM). Sin embargo, los estudios enumerados anteriormente sobre las dificultades en el aprendizaje de la lectura o de las matemáticas señalan que podría haber diferencias en los diferentes componentes del modelo entre los diversos trastornos. Así, en este estudio se consideró necesario analizar comparativamente la manera en que los distintos componentes de la memoria operativa, desde el modelo de Baddeley y Hitch (1974), están afectados en los niños que presentan diversos tipos de TEA. El objetivo de esta investigación, por lo tanto, consistió en examinar los perfiles de fortalezas y debilidades en estos tres componentes del funcionamiento de la memoria operativa en niños con diferentes TEA. Al comparar y contrastar los perfiles de funcionamiento de la memoria operativa en los diferentes TEA se buscó contribuir a la explicación de los hallazgos reportados en las relaciones entre varios tipos de Trastornos en el Aprendizaje.

\section{MÉTODO}

\section{Sujetos}

En el presente estudio se conformaron tres muestras no probabilísticas con niños de ambos sexos que estaban cursando segundo grado o terminando su primer año de primaria. Los niños provenían de tres colegios (uno masculino, uno femenino y uno mixto) de clase media (estratos socio-económicos 2 a 4 ) de la zona urbana de la ciudad de Cali, Colombia. Los niños sin TEA, con desempeño académico dentro del promedio tanto en lectura como en matemáticas, fueron escogidos de manera aleatoria entre aquellos cuyos padres asistieron a una reunión informativa previa. A partir del grupo de niños cuyos maestros consideraron que tenían algún TEA se hicieron las clasificaciones iniciales de los grupos TAL o TAL/TAM. Cada grupo estuvo constituido por 15 participantes de la siguiente manera: El grupo sin TEA se conformó con nueve niños y seis niñas, al igual que el grupo con TAL. El grupo con TAL/ TAM estuvo compuesto por siete niños y ocho niñas. La edad de los niños que participaron en el estudio estuvo entre 
siete años, cero meses y ocho años, cuatro meses de edad. Se realizó un ANOVA con el fin de comparar la edad de los tres grupos incluidos en la investigación, encontrando que no había un efecto de grupo $\left(\mathrm{F}_{2,42}=0,15, \mathrm{p}=0,86\right)$, lo cual señala que la edad de los sujetos no mostraba diferencias entre ellos. Todos los niños que participaron en el estudio presentaban un nivel de inteligencia dentro del promedio, no tenían historia de trastornos emocionales o neurológicos, de trastorno por déficit de atención e hiperactividad (TDAH), de trastorno de lenguaje ni de problemas sensoriales como limitaciones visuales o auditivas.

Los padres de todos los niños que participaron en la investigación fueron informados del proceso de la investigación, y firmaron un consentimiento informado.

\section{INSTRUMENTOS}

Instrumentos empleados para determinar la inclusión en los grupos de la investigación

La presencia o ausencia de trastornos emocionales o neurológicos, de trastornos de lenguaje, o de limitaciones sensoriales se realizó por medio de una entrevista estructurada a los padres de familia, complementada por una observación clínica. La posible presencia de TDAH se evaluó por medio de una escala que utiliza los criterios diagnósticos del DSM-IV (Escala de Evaluación de los Trastornos de la Conducta Perturbadora; Barkley, 1997), la cual fue contestada tanto por padres como por maestros.
Con el fin de estimar las habilidades intelectuales generales de los niños, se utilizaron las subpruebas de Semejanzas y Matrices del WISC-IV, estandarización mexicana (Wechsler, 2007). Se escogieron estas dos subpruebas con el fin de contar con una de razonamiento verbal y otra de razonamiento perceptual, y por ser las que mayor correlación tienen con el CI total para la edad escogida para esta investigación.

\section{Instrumentos empleados para determinar la conformación} de los grupos de la investigación

Una vez se determinó que un niño cumplía con los criterios para ser incluido en la investigación, se aplicaron las subpruebas de Lectura y de Aritmética de la Evaluación Neuropsicológica Infantil (ENI; Matute, Rosselli, Ardila \& Ostrosky-Solis, 2007) con el fin de establecer el grupo al que pertenecía dentro del estudio. Los niños que tenían un desempeño igual o menor a un puntaje estándar de 85 en lectura, especialmente en Precisión y Velocidad, formaron el grupo de TAL; el grupo de TAL/TAM estuvo compuesto por niños que tenían un desempeño igual o menor a un puntaje estándar de 85, tanto en lectura como en aritmética; por último, los niños del grupo control (Sin TEA) fue conformado por niños con un desempeño dentro del promedio (puntaje estándar entre 90 y 110), tanto en lectura como en aritmética. En la Tabla 1 se presentan las medias y desviaciones estándar de las diferentes medidas de lectura y aritmética de la ENI por grupo, sin TEA, TAL y TAL/TAM

Tabla 1.

Medias y Desviaciones estándar de las Medidas de Desempeño Académico (Lectura y Aritmética de la ENI) por Grupo (Sin TEA, TAL y TAL/TAM)

\begin{tabular}{|c|c|c|c|c|c|c|c|c|c|}
\hline & \multicolumn{3}{|c|}{ Sin TEA } & \multicolumn{3}{|c|}{ TAL } & \multicolumn{3}{|c|}{ TAL/TAM } \\
\hline & $\mathbf{N}$ & Media & DS & $\mathbf{N}$ & Media & DS & $\mathbf{N}$ & Media & DS \\
\hline \multicolumn{10}{|c|}{ Pruebas de Lectura } \\
\hline Precisión & 15 & 106,67 & 6,73 & 15 & 79,33 & 6,51 & 15 & 62,00 & 14,61 \\
\hline Comprensión & 15 & 111,67 & 11,44 & 15 & 90,53 & 13,04 & 15 & 85,80 & 12,95 \\
\hline Velocidad & 15 & 94,20 & 6,63 & 15 & 77,53 & 11,43 & 15 & 65,33 & 17,06 \\
\hline \multicolumn{10}{|c|}{ Pruebas de Aritmética } \\
\hline Conteo & 15 & 106,00 & 13,26 & 15 & 101,33 & 13,29 & 15 & 91,33 & 15,64 \\
\hline Manejo Numérico & 15 & 112,33 & 9,23 & 15 & 103,67 & 10,60 & 15 & 79,87 & 12,08 \\
\hline Cálculo & 15 & 108,53 & 9,75 & 15 & 100,53 & 8,11 & 15 & 80,00 & 9,06 \\
\hline Problemas Aritméticos & 15 & 101,67 & 9,00 & 15 & 96,67 & 5,23 & 15 & 91,67 & 16,22 \\
\hline
\end{tabular}

Nota. Sin TEA = Sin trastorno específico en el aprendizaje; TAL = Trastorno en el aprendizaje de la lectura; TAL/TAM = Trastorno concomitante en el aprendizaje 
Instrumentos para evaluar la memoria operativa

Para determinar el perfil de memoria operativa de cada uno de los grupos de la investigación se utilizó la Automated Working Memory Assessment [AWMA por sus siglas en inglés] (Alloway, 2007), versión experimental en español. Esta prueba es computarizada y permite evaluar los tres componentes básicos de memoria operativa propuestos por Baddeley y Hitch (1974): el bucle fonológico, la agenda viso-espacial y el EC. Para valorar el EC, la AWMA cuenta con tareas que requieren de procesamiento y almacenamiento simultáneo, tanto de información verbal como viso-espacial. Cada subprueba comienza con una serie de ítems de práctica, seguidos por los ítems del test.

Las subpruebas del AWMA que permiten evaluar el bucle fonológico o la MCP verbal son: Retención Directa de Dígitos, Retención de Palabras y Retención de NoPalabras. Las que buscan medir el funcionamiento de la agenda viso-espacial o la MCP viso-espacial son: Matriz de Puntos, Memoria de Laberintos y Recuerdo de Bloques. Para valorar la memoria operativa verbal (EC con información verbal) se incluyen las siguientes subrpruebas: Span de Escucha, Span de Conteo y Retención Inversa de Dígitos. Por último, las medidas que evalúan la memoria operativa viso-espacial (EC con información viso-espacial) son: Elemento Extraño, Señor X y Span Espacial. Tanto Alloway (2007) como Alloway, Gathercole \& Pickering (2006) hacen una descripción detallada de todas estas medidas.

\section{Análisis de los datos}

Se utilizó el programa de análisis estadístico PASW Statistics 18 para comparar los tres grupos de niños en los puntajes de las medidas de memoria operativa. Se realizaron análisis de varianza unidireccional (ANOVA) con el fin de evaluar si las medias de los grupos de niños en las distintas medidas difieren de manera significativa. Posteriormente, se hicieron comparaciones post-hoc con la prueba de Bonferroni para determinar la significancia de estas diferencias entre los diferentes grupos incluidos en la investigación: sin TEA, TAL y TAL/TAM.

\section{RESULTADOS}

La comparación de medias de las medidas de memoria operativa del AWMA entre los tres grupos se presenta en la Tabla 1. Adicionalmente, la figura 1 muestra la proporción de respuestas correctas de los tres grupos (Sin TEA, TAL y TAL/TAM) en las medidas de memoria operativa del AWMA. Tanto la tabla como el gráfico muestran que en la gran mayoría de las subpruebas el grupo sin TEA presentó los mejores desempeños, seguido por el grupo con TAL, siendo el grupo con TAL/TAM el que presentó los desempeños más bajos. Los ANOVAs realizados mostraron un efecto significativo de grupo en todas las medidas, como se puede observar en la tabla 2.

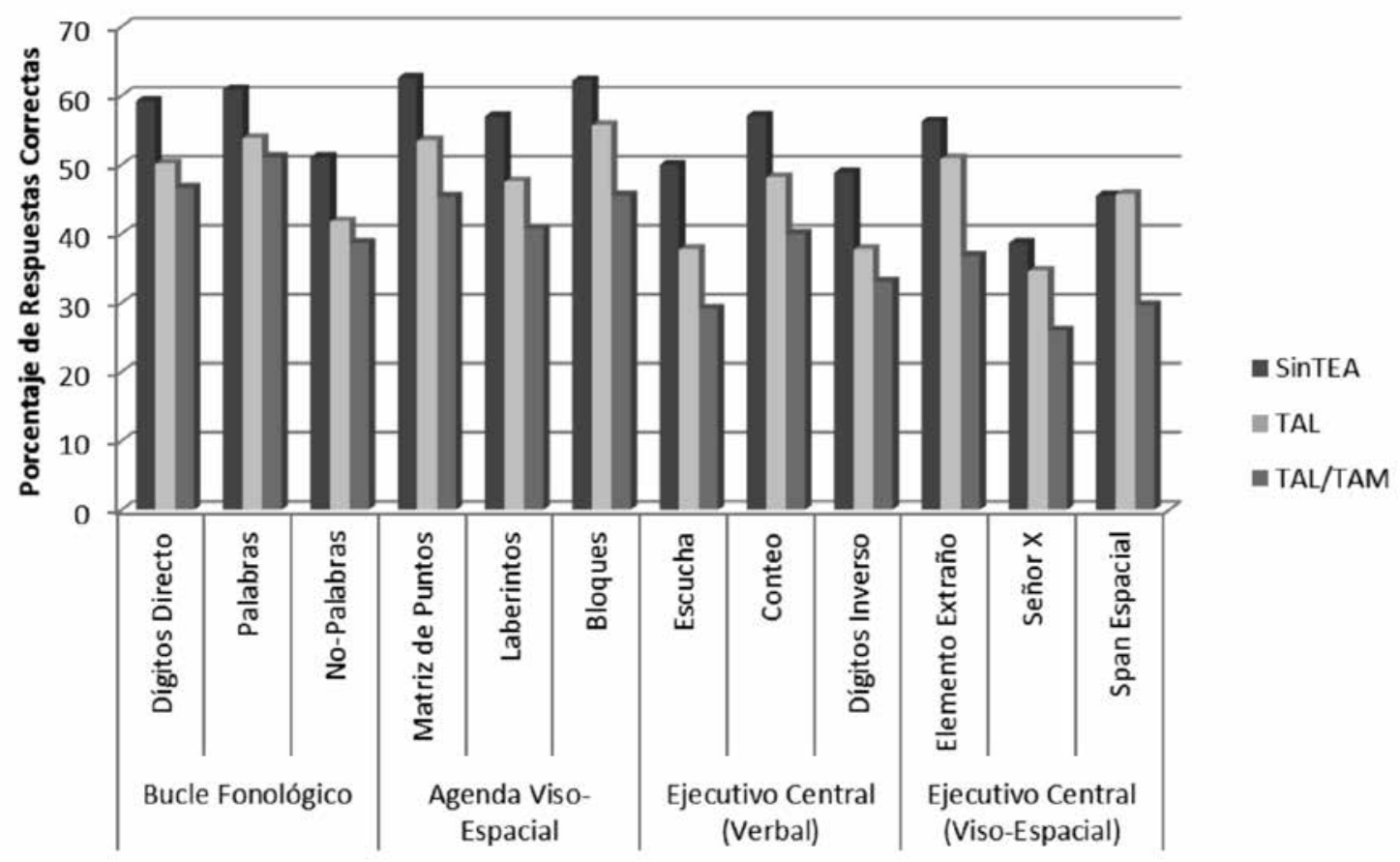

Figura1. Proporción de respuestas correctas de los tres grupos (Sin TEA, TAL y TAL/TAM) en las medidas de memoria operativa del AWMA 
Tabla 2.

Comparación de Medias de las Medidas de Memoria Operativa del AWMA por Grupo (Sin TEA, TAL y TAL/TAM)

\begin{tabular}{|c|c|c|c|c|c|c|c|c|c|c|}
\hline & \multicolumn{3}{|c|}{ Sin TEA } & \multicolumn{3}{|c|}{ TAL } & \multicolumn{3}{|c|}{ TAL/TAM } & \multirow{2}{*}{$\begin{array}{c}\text { Estadístico } \\
\mathrm{F}(2,42) \\
\end{array}$} \\
\hline & $\mathbf{N}$ & Media & DS & $\mathbf{N}$ & Media & DS & $\mathbf{N}$ & Media & DS & \\
\hline \multicolumn{11}{|c|}{ Bucle Fonológico } \\
\hline $\begin{array}{l}\text { Retención Directa de } \\
\text { Dígitos }\end{array}$ & 15 & 24,87 & 4,17 & 15 & 21,07 & 3,58 & 15 & 19,60 & 2,92 & $8,58^{* *}$ \\
\hline Retención de Palabras & 15 & 21,93 & 2,94 & 15 & 19,40 & 3,42 & 15 & 18,40 & 2,80 & $5,30^{* *}$ \\
\hline Retención de No-Palabras & 15 & 15,33 & 3,70 & 15 & 12,53 & 4,52 & 15 & 11,60 & 2,13 & $4,40^{*}$ \\
\hline \multicolumn{11}{|c|}{ Agenda Viso-Espacial } \\
\hline Matriz de Puntos & 15 & 22,53 & 3,93 & 15 & 19,27 & 2,66 & 15 & 16,33 & 3,79 & $11,74^{* * *}$ \\
\hline Memoria de Laberintos & 15 & 20,53 & 4,87 & 15 & 17,13 & 3,20 & 15 & 14,67 & 6,26 & $5,33^{* *}$ \\
\hline Span de Bloques & 15 & 22,40 & 3,25 & 15 & 20,07 & 2,96 & 15 & 16,40 & 3,31 & $13,59^{* * *}$ \\
\hline \multicolumn{11}{|c|}{ Ejecutivo Central - Verbal } \\
\hline Span de Escucha & 15 & 12,00 & 2,30 & 15 & 9,07 & 3,41 & 15 & 7,00 & 3,63 & $9,45^{* * *}$ \\
\hline Span de Conteo & 15 & 17,13 & 3,23 & 15 & 14,47 & 3,68 & 15 & 12,00 & 2,78 & $9,36^{* * *}$ \\
\hline $\begin{array}{l}\text { Retención Inversa de } \\
\text { Dígitos }\end{array}$ & 15 & 11,73 & 3,26 & 15 & 9,07 & 2,84 & 15 & 7,93 & 2,31 & $7,12^{* *}$ \\
\hline \multicolumn{11}{|c|}{ Ejecutivo Central - Viso-Espacial } \\
\hline Elemento Extraño & 15 & 20,27 & 4,10 & 15 & 18,33 & 3,16 & 15 & 13,27 & 3,62 & $14,78^{* * *}$ \\
\hline Señor $X$ & 15 & 11,60 & 3,96 & 15 & 10,40 & 2,41 & 15 & 7,80 & 3,08 & $5,48^{* *}$ \\
\hline Span Espacial & 15 & 16,40 & 4,15 & 15 & 16,47 & 5,22 & 15 & 10,67 & 4,01 & $3,66^{* *}$ \\
\hline
\end{tabular}

Nota. Sin TEA = Sin trastorno específico en el aprendizaje; TAL = Trastorno en el aprendizaje de la lectura; TAL/TAM = Trastorno concomitante en el aprendizaje de la lectura y de las matemáticas

$* * * \mathrm{p}<0,001 \quad * * \mathrm{p}<0,01 \quad * \mathrm{p}<0,05$

Las comparaciones post-hoc con la prueba de Bonferroni $(\mathrm{p}<0,05)$ en las subpruebas que valoran el bucle fonológico evidenciaron que las medias de los dos grupos con TEA (TAL y TAL/TAM) fueron significativamente más bajas que la del grupo sin TEA en Retención Directa de Dígitos ( $\operatorname{Sin}$ TEA $>$ TAL, $p=0,02$ y Sin TEA $>$ TAL/TAM, $p=0,001$ ), mientras que los dos grupos con TEA no difirieron entre sí $(\mathrm{p}=0,81)$. En Retención de Palabras y Retención de No-Palabras la única diferencia significativa se presenta entre el grupo sin TEA y el grupo con TAL/TAM (Retención de Palabras, $\mathrm{p}=0,009$ y Retención de No-Palabras, $\mathrm{p}=0,02$ ), presentando este último el desempeño más bajo en ambas subpruebas.
En las medidas utilizadas para evaluar el componente de Agenda Viso-Espacial se observó en las pruebas post-hoc que en Matriz de Puntos, la media del grupo sin TEA es significativamente mayor a la de los dos grupos con TEA ( Sin TEA $>$ TAL, $\mathrm{p}=0,04$ y $\sin$ TEA $>$ TAL/TAM, $\mathrm{p}<0,001$ ), los cuales no difirieron de manera significativa entre sí $(\mathrm{p}=0,08)$. En Memoria de Laberintos sólo se presentó una diferencia significativa entre el grupo sin TEA y el grupo con TAL/TAM ( $p=0,007)$. En Span de Bloques el grupo con TAL/TAM mostró un resultado significativamente inferior respecto a los grupos sin TEA y TAL (Sin TEA $>$ TAL/ TAM, $p<0,001$ y TAL $>$ TAL/TAM, $p=0,009)$, los cuales no presentaron diferencias entre sí $(\mathrm{p}=0,15)$. 
En las tareas de Ejecutivo Central con información verbal las pruebas post-hoc mostraron que en Span de Escucha y en Retención Inversa de Dígitos se presentaron diferencias significativas entre el grupo sin TEA y los dos grupos con TEA, obteniendo el grupo sin TEA medias superiores al grupo con TAL, en Span de Escucha $(\mathrm{p}=0,045)$ y en Retención Inversa de Dígitos $(\mathrm{p}=0,04)$. El grupo sin TEA también alcanzó medias superiores que el grupo con TAL/TAM en Span de Escucha $(\mathrm{p}<0,001)$ y en Retención Inversa de Dígitos $(\mathrm{p}=0,002)$. A su vez, estos grupos no difirieron entre sí ni en Span de Escucha $(\mathrm{p}=0,24)$ ni en Retención Inversa de Dígitos $(\mathrm{p}=0,04)$. En Span de Conteo se presentó una diferencia significativa entre el grupo sin TEA y el grupo con TAL/TAM ( $<<0,001)$, en donde el grupo sin TEA tuvo el mejor desempeño. En esta tarea no se presentaron diferencias significativas entre el grupo sin TEA y el grupo con TAL $(p=0,09)$ ni entre el grupo con TAL y el grupo con TAL/TAM $(\mathrm{p}=0,13)$.

Finalmente, el análisis post-hoc de las subpruebas utilizadas para medir el funcionamiento del Ejecutivo Central con información viso-espacial mostró que en dos de estas medidas (Elemento Extraño y Span Espacial) el grupo con TAL/TAM difirió significativamente del grupo sin TEA(Sin TEA $>$ TAL/TAM en Elemento Extraño, $p<0,001$ y en Span Espacial, $\mathrm{p}=0,003)$. Por otra parte, el grupo con TAL/TAM también presentó diferencias significativas respecto al grupo con TAL (TAL > TAL/TAM en Elemento Extraño $p=0,001$ y en Span Espacial, $\mathrm{p}=0,003)$. Entre ambos grupos con trastornos en el aprendizaje no se presentaron diferencias significativas en estas dos subpruebas ( $\sin$ TEA vs. TAL en Elemento Extraño, $p=0,46$ y en Span Espacial, $p=1,00$ ). En la subprueba de Señor X, sólo se presentó una diferencia significativa entre el grupo sin TEA y el grupo con TAL/ TAM ( $\mathrm{p}=0,007)$, el cual presentó los desempeños más bajos.

De acuerdo con lo anterior, se evidencia que los niños con TAL se diferencian de los niños sin TEA principalmente en tareas de EC con información verbal. Estos dos grupos de niños no se diferencian en tareas de EC con información viso-espacial. Por otro lado, los niños con la doble condición de trastorno en el aprendizaje, TAL/TAM, presentan diferencias significativas respecto a los niños sin TEA en todas las medidas del AWMA. Al comparar los niños con TAL y los que presentan TAL/TAM, se puede observar que se diferencian entre sí en la ejecución de algunas tareas de tipo viso-espacial (Span de Bloques, Elemento Extraño y Span Espacial).

\section{DISCUSIÓN}

La presente investigación buscaba explorar el funcionamiento de las habilidades de memoria operativa en niños con trastornos en el aprendizaje y determinar si los hallazgos de investigaciones similares en otras lenguas, principalmente en inglés, se pueden generalizar a niños hispano-parlantes. Este es uno de los pocos trabajos en español que han abordado el estudio de la memoria operativa en estos niños. Igualmente, este estudio buscaba comparar el funcionamiento de la memoria operativa en varios tipos de trastornos específicos en el aprendizaje, de tal manera que se hiciera un aporte a la explicación de los hallazgos reportados en la literatura sobre las relaciones cognoscitivas entre dichos trastornos.

Al igual que algunos estudios previos (Baqués \& Sáiz, 1999; Pickering, 2006; Stone \& Brady, 1995), la presente investigación muestra que los niños con trastornos en el aprendizaje de la lectura (TAL y TAL/TAM) presentan un déficit en el componente del Bucle Fonológico del modelo de memoria operativa de Baddley y Hitch (1974), aunque el grupo con TAL sólo evidenció un déficit significativo en Retención Directa de Dígitos.

Aunque el grupo con TAL obtuvo una media inferior al grupo sin TEA en Retención de No-Palabras, el desempeño en esta prueba no evidenció un déficit significativo en el grupo de niños con TAL. En otras palabras, los puntajes de los niños con TAL en Retención de No-Palabras no evidenciaron diferencias estadísticamente significativas respecto a los puntajes de los niños sin TEA en esta prueba. Este hallazgo no es consistente con estudios previos que muestran que la repetición de pseudopalabras es significativamente inferior en niños con TAL debido a las dificultades relacionadas con la decodificación lectora cuando se utiliza la ruta fonológica para una adecuada conversión grafema-fonema. En general, se ha considerado que este tipo de pruebas contribuye en la evaluación de las representaciones fonológicas requeridas para el aprendizaje de la lectura (De Jong \& Olson, 2004; Pickering, 2006; Stone \& Brady, 1995).

Sin embargo, en varios de estos estudios se muestra que esta asociación entre la memoria de no-palabras y TAL a veces no es evidente durante las primeras etapas del aprendizaje de la lectura, sino que aparece en niños mayores, a partir de los ocho o nueve años debido a que su progreso en la construcción de representaciones fonológicas es más lento que en los lectores normales, como se evidencia en algunos estudios longitudinales (Mutter \& Snowling, 1998; Snowling, Goulandris \& Defty, 1996). Ya en el presente estudio se observa una diferencia en la Retención de NoPalabras entre los niños sin TEA y los niños con TAL que posiblemente aumente con los años, como se ha visto en los estudios citados anteriormente.

Adicionalmente, es importante anotar que existen diferentes tipos de pruebas para medir la memoria de no-palabras; en varios de los estudios revisados la prueba utilizada fue la 
de Repetición de No-Palabras (Gathercole \& Baddeley,1989) en la cual los niños deben repetir pseudopalabras cuyo número de sílabas se va incrementando, utilizando el sistema fonético del inglés (por ejemplo, tin, manpit, tonpunner, etc.). Por su parte, en la presente investigación se aplicó una prueba de Retención de No-Palabras que utiliza un esquema similar al de Retención Directa de Dígitos. Así, se presentan pseudopalabras de una o dos sílabas simples siguiendo el esquema fonético del español (por ejemplo, sila, monu-pede, dica-pento-roti, etc.). Estas diferencias, tanto de la lengua como del tipo de prueba utilizada en las investigaciones, podrían estar determinando que no se encuentre un déficit significativo en los niños hispano hablantes con TAL en este estudio. Por lo tanto, es necesario explorar la relación entre la memoria de pseudopalabras y el aprendizaje de la decodificación lectora en español en niños de diferentes edades.

Los resultados en las pruebas del AWMA que miden el Ejecutivo Central con información verbal en los niños con TAL presentaron diferencias significativas respecto a los niños sin TEA en dos de las tres pruebas: Span de Escucha y Retención Inversa de Dígitos. Los niños con TAL/TAM, por su lado, evidenciaron diferencias significativas en todas las pruebas que valoran esta habilidad cognoscitiva. Lo anterior confirma los resultados de un gran número de estudios en niños con dificultades en lectura y/o matemáticas, los cuales establecen una alta relación del Ejecutivo Central con información verbal y aprendizaje escolar (Alsina, 2007; Baqués \& Sáiz, 1999; Gathercole et al., 2006; Geary et al., 2007; Passolunghi \& Siegel, 2004; Pickering, 2006; Swanson, 2003).

Es importante anotar que aunque el grupo con TAL tuvo un desempeño inferior al grupo sin TEA en el Span de Conteo, éste sólo fue significativamente inferior en el grupo con TAL/TAM, al tiempo que los dos grupos con TEA no mostraron diferencias entre sí. Esto indica que esta medida tiene más relación con las dificultades en matemáticas que con las de lectura. Otras investigaciones reportadas en la literatura ya han señalado que los niños con dificultades en las matemáticas presentan más déficit en pruebas de memoria operativa con información numérica, en especial en tareas similares a las de Span de Conteo de la presente investigación (Bull \& Scerif, 2001; Geary, Hoard, Byrd-Craven \& DeSoto, 2004; Miranda-Casas et al., 2006; Passolunghi \& Siegel, 2004).

Las medidas para valorar el componente de la Agenda Viso-Espacial mostraron que los niños con TAL/TAM presentaban desempeños inferiores a los niños sin TEA en todas las tareas. Estos niños, además, evidenciaron un desempeño inferior a los niños con TAL en Span de Bloques, prueba en la que los niños con TAL no se diferenciaron de los niños sin TEA. Estos resultados muestran que los niños que presentaban dificultades significativas en ambas áreas académicas, lectura y aritmética, exhibían mayores déficits en este componente de la memoria operativa que quienes sólo tenían dificultades en la lectura. Estudios reportados por Pickering (2006) han mostrado que los niños con TAL no presentan déficits en la agenda viso-espacial, lo cual se confirma parcialmente en esta investigación, puesto que estos niños no difirieron de los niños sin TEA en dos de las tres medidas utilizadas: Memoria de Laberintos y Span de Bloques.

Adicionalmente, algunos estudios previos con niños que presentaban un desempeño por debajo del promedio en aritmética, no han encontrado un déficit en el componente de agenda viso-espacial de la memoria operativa (Alsina \& Sáiz-Roca, 2003; Bull, Johnston \& Roy, 1999; Geary et al., 2007). Sin embargo, las investigaciones en que se incluyeron niños con trastorno en el aprendizaje de las matemáticas sí mostraron un bajo nivel en el desempeño de este tipo de tareas (Geary et al., 2007; McClean \& Hitch, 1999; Miranda-Casas et al., 2006). La presente investigación confirma lo hallado en estos últimos estudios citados, estableciendo una relación significativa entre el desempeño en aritmética y el componente de Agenda Viso-Espacial.

En este estudio se utilizaron medidas que permiten evaluar el funcionamiento del Ejecutivo Central con información viso-espacial, las cuales sólo recientemente se han desarrollado, permitiendo evaluar la ejecución en tareas viso-espaciales que requieren tanto de procesamiento de la información como del almacenamiento de ésta. Por este motivo, en la revisión bibliográfica realizada no se encontraron investigaciones previas que utilicen este tipo de medidas. El presente estudio confirma la tendencia de los niños con TAL/TAM a presentar un déficit significativo en la memoria operativa viso-espacial respecto a los niños sin TEA en las tres medidas empleadas y respecto a los niños con TAL en dos de éstas, Elemento Extraño y Span Espacial. También se muestra nuevamente que los niños con TAL no presentan diferencias en tareas viso-espaciales respecto a los niños sin TEA.

Lo anterior lleva a concluir que los niños con TAL, con o sin dificultades significativas en matemáticas, evidencian grandes dificultades en la memoria operativa verbal cuando ésta requiere tanto de procesamiento como de almacenamiento de la información verbal, excepto cuando la información a procesar es numérica. Sin embargo, cuando no presentan dificultades en matemáticas, estos niños pueden no evidenciar un déficit significativo tanto en el componente de agenda viso-espacial del modelo de memoria operativa de Baddeley y Hitch (1974) como en el ejecutivo central con información viso-espacial. Por otro 
lado, los niños con TAL/TAM sí presentan déficits en la memoria operativa verbal con información numérica y en la memoria operativa viso-espacial, situándose esta última por debajo de la de los niños con TAL, en especial cuando se requiere no sólo de procesamiento de la información, sino también del almacenamiento de ésta. Muy posiblemente, este déficit en la memoria operativa verbal con información numérica y en la memoria operativa viso-espacial sea lo que pone en riesgo a los niños que ya presentan limitaciones en su aprendizaje de la lectura, para presentar dificultades significativas adicionales en matemáticas.

Retomando los objetivos de la presente investigación, se puede concluir que los niños con TAL se diferencian de los niños sin TEA principalmente en tareas de EC con información verbal, específicamente en aquellas que requieren de procesamiento complejo de la información, más allá del recuerdo pasivo de ésta. Contrariamente, estos dos grupos de niños no se diferencian en tareas de EC con información viso-espacial. Por otro lado, los niños con la doble condición de trastorno en el aprendizaje, TAL/TAM, presentan diferencias significativas respecto a los niños sin TEA en todas las medidas del AWMA, incluyendo aquéllas que miden procesamiento y almacenamiento de información verbal (Bucle Fonológico y EC con información verbal) y las que miden la memoria operativa viso-espacial (Agenda Viso-Espacial y EC con información viso-espacial). Al comparar los niños con TAL y los que presentan TAL/ TAM, se pudo determinar que, en esencia, se diferencian entre sí en la ejecución de tareas de tipo viso-espacial, especialmente cuando se requiere procesamiento complejo de la información.

Al tratar de determinar el perfil de fortalezas y debilidades en la memoria operativa de los niños con trastornos en el aprendizaje de este estudio, se puede concluir que los niños con TAL presentan una fortaleza en la memoria operativa viso-espacial y una debilidad en la memoria operativa verbal que exige, tanto almacenamiento como procesamiento complejo de la información, en especial cuando la información es lingüística y no numérica. Los niños con TAL/TAM presentan una debilidad significativa en todas las tareas de memoria operativa medidas por el AWMA. Esto lleva a suponer que son las dificultades en el procesamiento y almacenamiento de la información verbal lo que origina riesgo en el desarrollo de problemas en lectura, mientras que el déficit en el procesamiento y almacenamiento de información viso-espacial añade un riesgo adicional para presentar dificultades en las matemáticas. De esta manera, se evidencia que los diversos componentes del modelo de memoria operativa de Baddeley y Hitch (1974) están alterados de manera diferente en los distintos tipos de TEA estudiados, al mismo tiempo que se evidencia la importancia del EC en el aprendizaje escolar.

La teoría evolutiva de Geary $(2003,2005,2007)$ resalta la importancia del rol de la memoria operativa en el aprendizaje escolar. Según el modelo de Geary, un déficit en este sistema cognoscitivo afectaría el aprendizaje académico, tanto de la lectura como de las matemáticas. La presente investigación confirma la importancia de esta habilidad neurocognoscitiva, resaltando que los niños con TEA presentan un déficit en el funcionamiento de la memoria operativa que se asume mayor cuando las dificultades en el aprendizaje son más generalizadas, como es el caso de los niños con TAL/TAM.

\section{REFERENCIAS}

Alsina, A. (2007). ¿Por qué algunos niños tienen dificultades para calcular? Una aproximación desde el estudio de la memoria humana. Revista Latinoamericana de Investigación en Matemática Educativa, 10(3), 315-333.

Alsina, A. \& Sáiz-Roca, D. (2003). Un análisis comparativo del papel del bucle fonológico versus la agenda viso-espacial en el cálculo en niños de 7-8 años. Psychothema, 15(2), 241-246.

Alloway, T. P. (2007). Automated working memory assessment. Londres: Pearson Assessment.

Alloway, T. P. \& Archibald, L. (2008). Working memory and learning in children with developmental coordination disorder and specific language impairment. Journal of Learning Disabilities, 41(3), 251-262.

Alloway, T. P., Gathercole, S. E, \& Pickering, S. J. (2006). Verbal and visuospatial short-term and working memory in children: Are they separable? Child Development, 77(6), 1698-1716.

Alloway, T. P. \& Temple, K. J. (2007). A comparison of working memory skills and learning in children with developmental coordination disorder and moderate learning difficulties. Applied Cognitive Psychology, 21(4), 473-487.

Alloway, T. P., Rajendran, G. \& Archibald, L. (2009). Working memory in children with developmental disorders. Journal of Learning Disabilities, 42(4), 372-382.

Baddeley, A. D. (2000). The episodic buffer: A new component of working memory? Trends in Cognitive Sciences, 4, 417423.

Baddeley, A. D. (2002). Is working memory still working? European Psychologist, 7(2), 85-97.

Baddeley, A. D. (2003). Working memory: Looking back and looking forward. Nature Reviews: Neuroscience, 4, 829-839.

Baddeley, A. D. \& Hitch, G. (1974). Working memory. En G. Bower (Ed.), The psychology of learning and motivation (Vol. 8, pp. 47-90). New York: Academic Press. 
Baddeley, A. D., \& Hitch, G. (1994). Developments in the concept of working memory. Neuropsychology, 4, 485-493.

Baqués, J. \& Sáiz, D. (1999). Medidas simples y compuestas de memoria de trabajo y su relación con el aprendizaje de la lectura. Psychothema, 11(4), 737-745.

Barkley, R. A. (1997). Niños desafiantes: Materiales de evaluación y folletos para los padres. New York: The Guilford Press.

Bull, R., Johnston, R. S. \& Roy, J. A. (1999). Exploring the roles of the visual-spatial sketchpad and central executive in children's arithmetical skills: Views from cognition and developmental neuropsychology. Developmental Neuropsychology, 15(3), 421-442.

Bull, R. \& Scerif, G. (2001). Executive functioning as predictor of children's mathematics ability: Inhibition, switching and working memory. Developmental Neuropsychology, 19(3), 273-293.

De Jong, P. F. \& Olson, R. K. (2004). Early predictors of letter knowledge. Journal of Experimental Child Psychology, 88, 254-273.

Gathercole, S. E., Alloway, T. P., Willis, C. \& Adams, A. M. (2006). Working memory in children with reading disabilities. Journal of Experimental Child Psychology, 93, 265281.

Gathercole, S. E. \& Baddeley, A. D. (1989). Evaluation of the role of phonological STM in the development of vocabulary in children: A longitudinal study. Journal of Memory and Language, 28(2), 200-213.

Geary, D. C. (2003). Evolución y desarrollo del conocimiento intuitivo: Implicaciones para el aprendizaje infantil. Infancia y Aprendizaje, 26(3), 287-308.

Geary, D. C. (2005). The origin of mind: Evolution of brain, cognition and general intelligence. Washington, DC: American Psychological Association.

Geary, D. C. (2007). An evolutionary perspective on learning disability in mathematics. Developmental Neuropsychology, 32(1), 1-49.

Geary, D. C., Hoard, M. K., Byrd-Craven, J. \& DeSoto, M. C. (2004). Strategy choices in simple and complex addition: Contributions of working memory and counting knowledge for children with mathematical disability. Journal of Experimental Child Psychology, 88, 121-151.

Geary, D. C., Hoard, M. K., Byrd-Craven, J., Nugent, L. \& Numtee, C. (2007). Cognitive mechanisms underlying achievement deficits in children with mathematical learning disability. Child Development, 78(4), 1343-1359.
Logie, R. H. (1995). Visuo-spatial working memory. Hove, East Sussex: Lawrence Erlbaum.

Matute, E., Roselli, M., Ardila, A. \& Ostrosky-Solís, F. (2007). Evaluación neuropsicológica infantil (ENI). México: El Manual Moderno.

McLean, J. F \& Hitch, G. J. (1999). Working memory impairments in children with specific arithmetic learning difficulties. Journal of Experimental Child Psychology, 74, 240-260.

Miranda-Casas, A., Meliá-de Alba, A., Marco-Taverner, R., Roselló, B. \& Mulas, F. (2006). Dificultades en el aprendizaje de matemáticas en niños con trastorno por déficit de atención e hiperactividad. Revista de Neurología, 42 (supl.2), S163-S170.

Mutter, V. \& Snowling, M. (1998). Concurrent and longitudinal predictors of reading: The role of metalinguistic and short-term memory skills. Reading Research Quarterly, 33(3), 320-337.

Passolunghi, M. C. \& Siegel, L. S. (2004). Working memory and access to numerical information in children with disability in mathematics. Journal of Experimental Child Psychology, 88, 348-367.

Pickering, S. J. (2006). Working memory in dyslexia. T. P. Alloway y S. E. Gathercole (Eds.), Working memory and neurodevelopmental disorders (pp.7-40). East Sussex: Psychology Press.

Pickering, S. J. \& Gathercole, S. E. (2004). Distinctive working memory profiles in children with special educational needs. Educational Psychology, 24(3), 393-408.

Sierra-Fitzgerald, O. \& Ocampo-Gaviria, T. (2013). El papel de la memoria operativa en las diferencias y trastornos del aprendizaje escolar. Revista Latinoamericana de Psicología, 45(1), 63-79.

Snowling, M. J., Goulandris, N. \& Defty, N. (1996). A longitudinal study of reading development in dyslexic children. Journal of Educational Psychology, 88(4), 653-669.

Stone, B. \& Brady, S. (1995). Evidence for phonological processing deficits in less-skilled readers. Annals of Dyslexia, $X L V, 51-78$.

Swanson, H. L. (2003). Age-related differences in learning disabled and skilled reader's working memory. Journal of Experimental Child Psychology, 85, 1-31.

Wechsler, D. (2007). WISC-IV: Escala Wechsler de inteligencia para niños- $I V$. México: El Manual Moderno. 